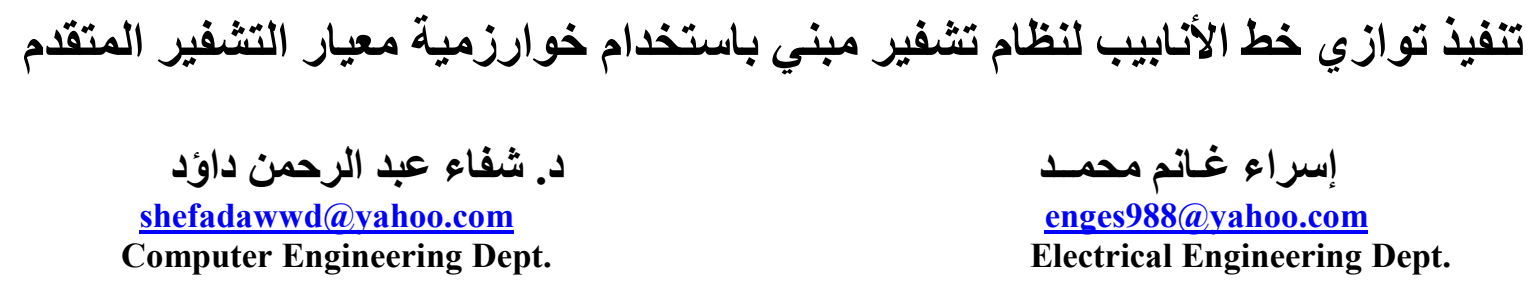

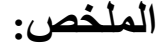

في هذا البحث تم تنفيذ خوارزمية الـ AES كونها معتمدة عالمياً في تثفير المعلومات لنظم الاتئ الاتصالات المختلفة أخذين

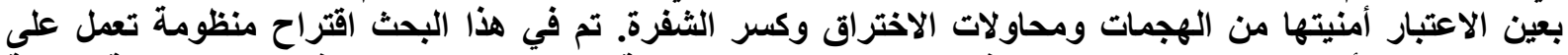

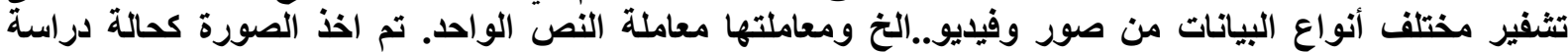

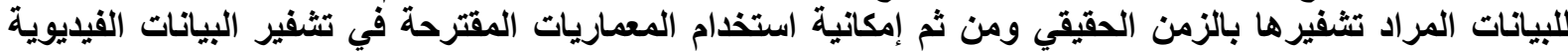

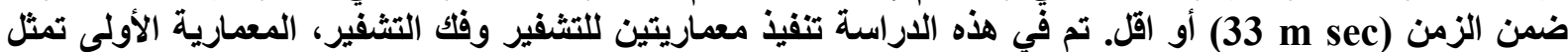

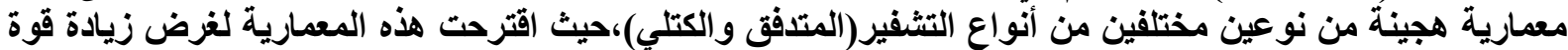

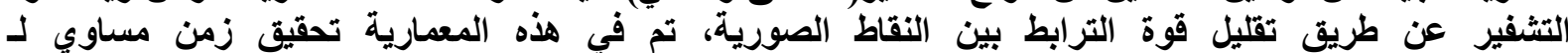

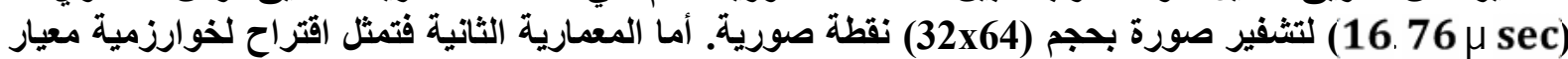

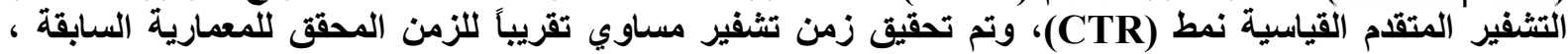

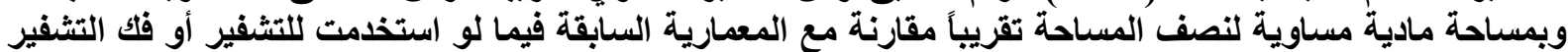

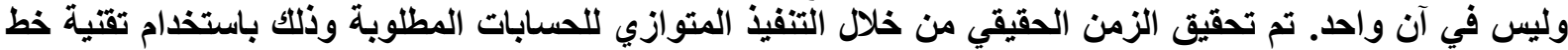

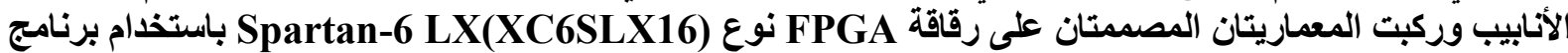

. ISE 14.2

\title{
Pipelined Parallel Implementation of Cryptosystems Based on Advanced Encryption Standard
}

\begin{abstract}
A hardware architecture implementation of Advanced Encryption Standard (AES) is globally adopted to encrypt data for variant communications systems, taking into account that AES is reliable, secured and immunized against attacks. A single crypto system is suggested to encrypt and/or decrypt different types of data .These types of data are assumed to be as a text data.The image is considered as a case study for the type of data that is to be encrypted in real time. Then the proposed architectures are used to encrypt the video within the time $\leq 33 \mathrm{~m}$ sec. Two architectures are proposed . The first one is a hybrid of both stream and block ciphering. This architecture is used to increase the encryption security by reducing the correlation among image pixels. The resulting encryption time for an image of (32x64) pixels is equal to $16.76 \mu \mathrm{sec}$. The second architecture is proposed for CTR mode of AES algorithm. The same time achieved in the first architecture is also achieved in this implementation. However, the half of the hardware resources in comparison with the first architecture is achieved in implementing the second, but if it is used for either encryption or decryption, not for both simultaneity. The real time implementation is achieved due to using parallel computation that is based on pipelining technique. The architecture are synthesized on Spartan-6 LX(XC6SLX16) using ISE 14.2 .
\end{abstract}

Keywords : AES , FPGA , Image Encryption , Pipeline design .

$$
\text { قبل: } 2014 \text { - } 3 \text { - } 6
$$

أستلم: 2013 - 11 - 11 - 17 
يجب أن تتمتع معظم البيانات التي يتم تداولها عبر وسائل الاتصال الحديث بخصوصية عالية للمستخدم والتي

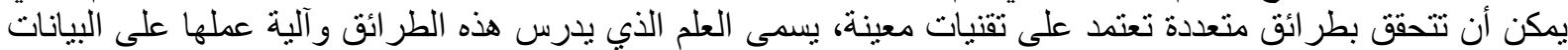

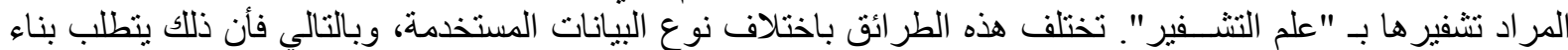

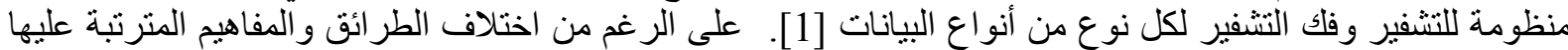

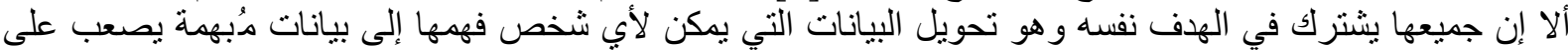

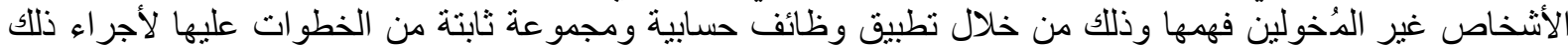

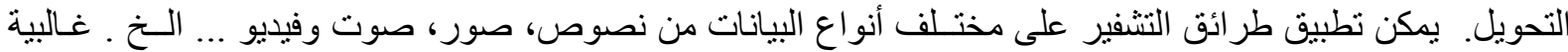

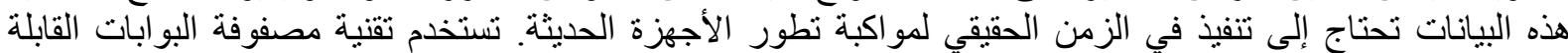

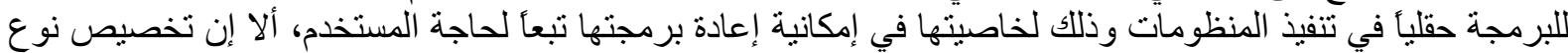

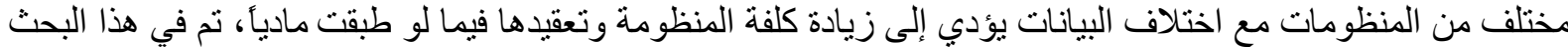

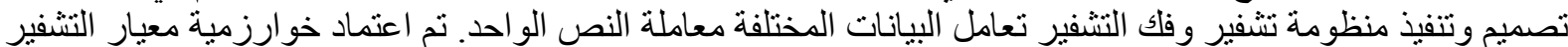

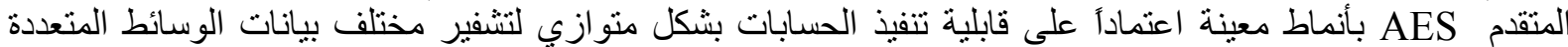
ومعاملتها معاملة النص. لتنفيذ هذه الأنماط وتحقيق منطلبات الزمن الحقيقي (

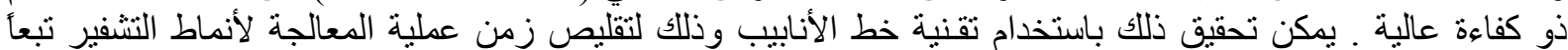

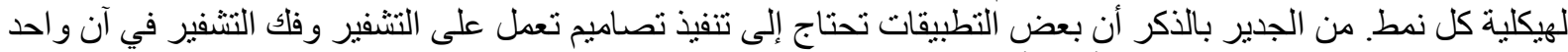

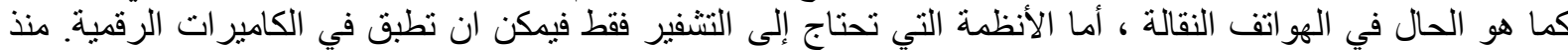

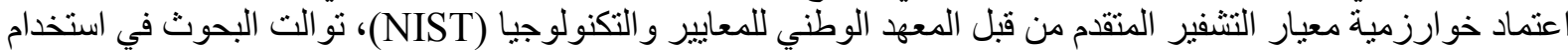

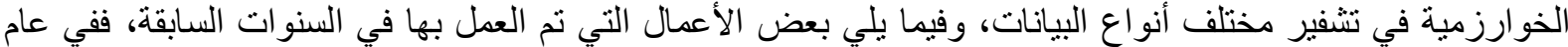

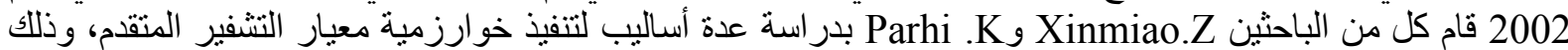

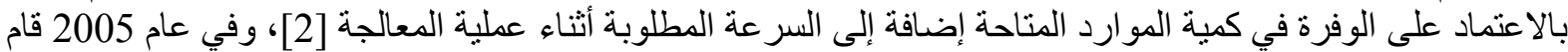

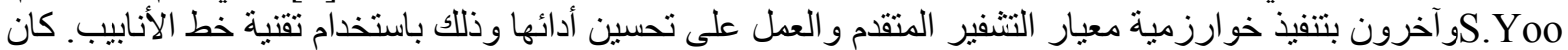

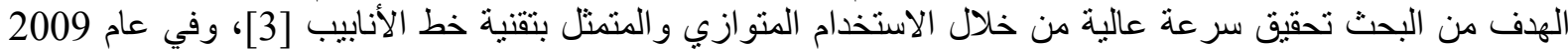

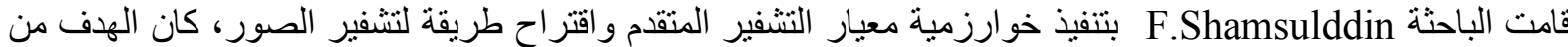

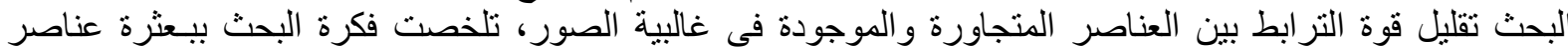

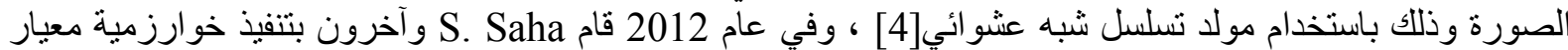

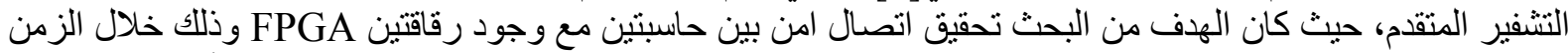

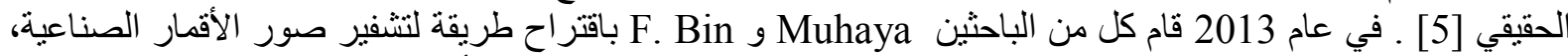

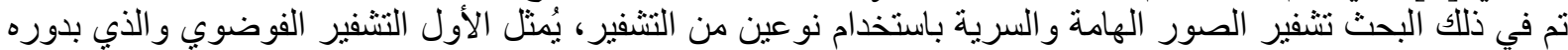

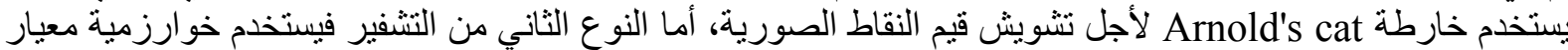

التشفير المتقدم للتشفير بشكل كتل للبيانات الصورية المشفرة التي تم الحصول عليها من النوع السابق من التشفير [6].

\section{2- 2 - 2 أنواع التثفير \\ يقسم التشفير بشكل عام إلى نوعين [7]:}

وفيه يكون مفتاح التشفير وفلك التشفير منماتثلان هذا النوع قد يتطلب قناة إضافية آمنة تعمل على نقل المفتاح

المتناظر بين الطرفين. يقسم التشفير المتناظر بدوره إلى نوعين، النوع الأول يقوم بتشفير البيانات على مستوى البـايت

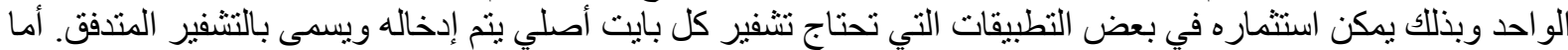

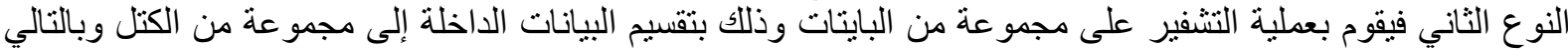

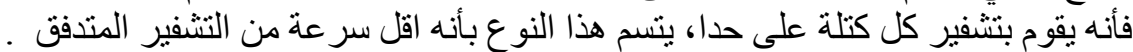

2.2

ويكون فيه مفتاح التشفير وفلك التشفير غير متمانثلان وهو بذللك لا يحتاج إلى قناة إضافية لنقل المفتاح يحتوي هذا

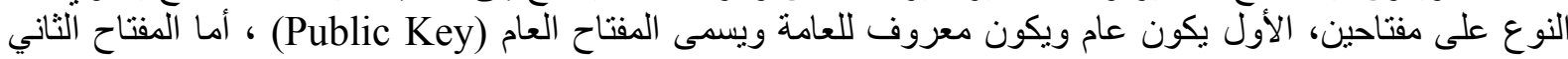
فيكون سري لا يعرفه سوى الثخص المخون عام ويكون ميسى بالمفتاح الخاص (Private Key) . 
3- هيكلية خوارزمية معيار التثفير المتقدم- ريجندال ( AES-Rijindal

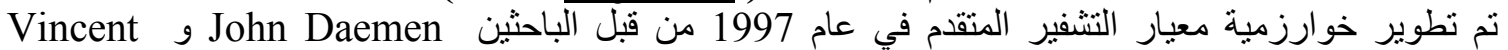

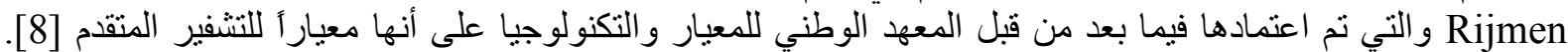

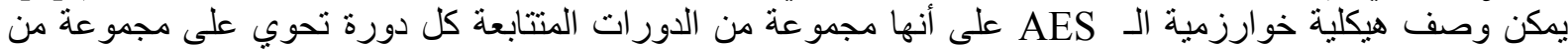

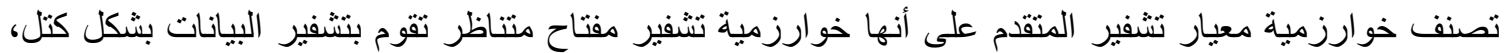
المر احل

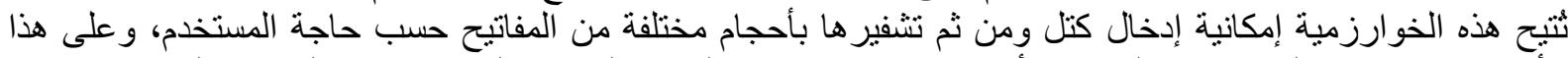

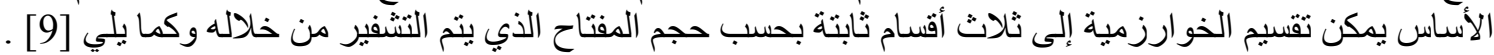

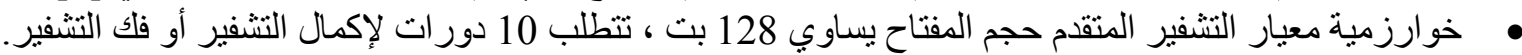

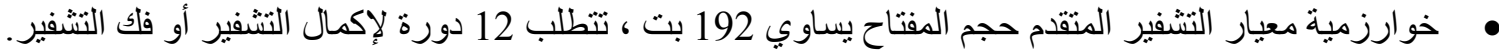

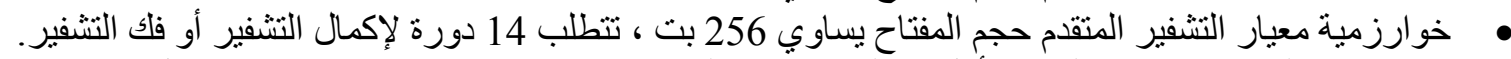

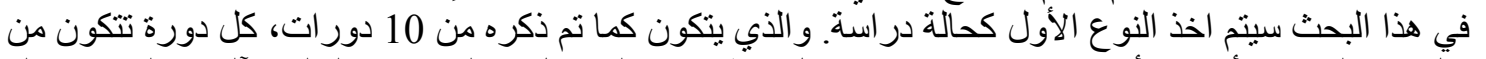

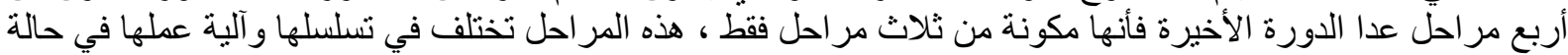

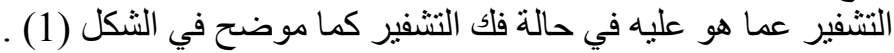

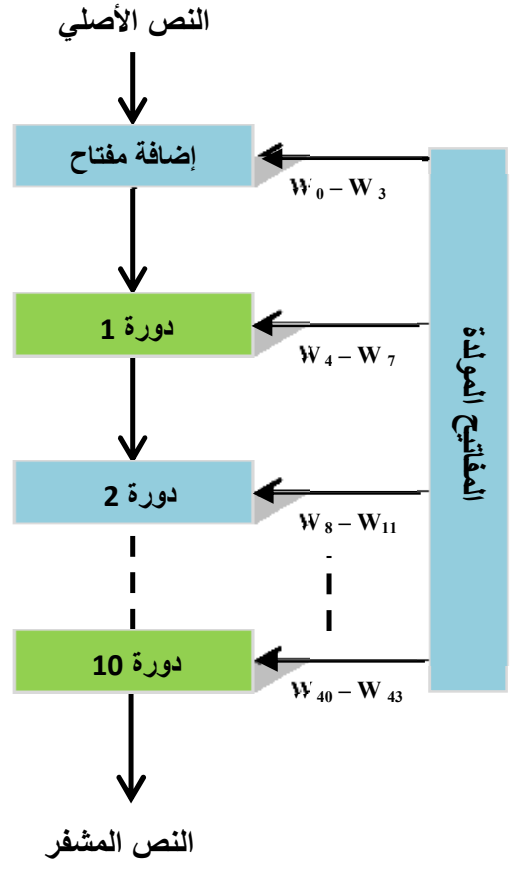

A

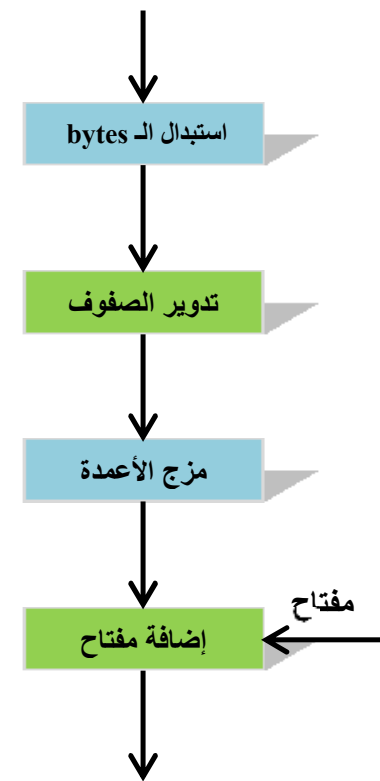

B

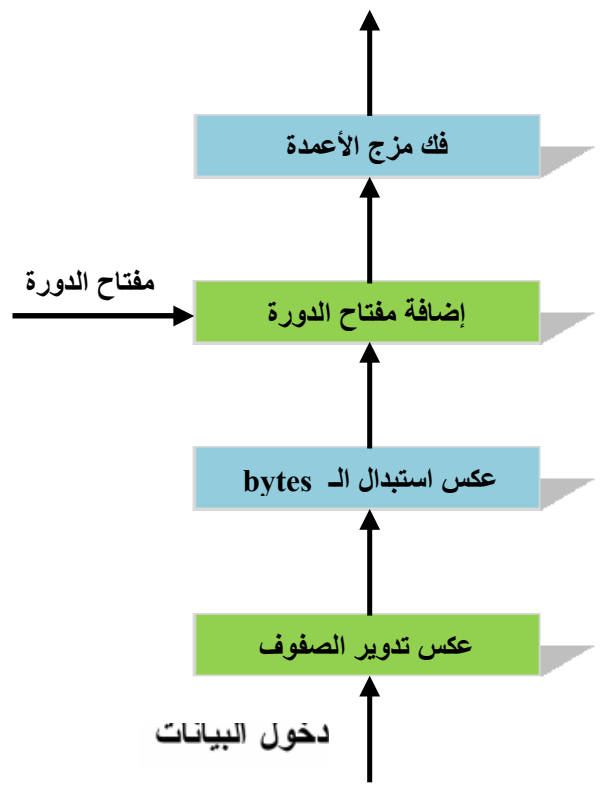

$\mathrm{C}$

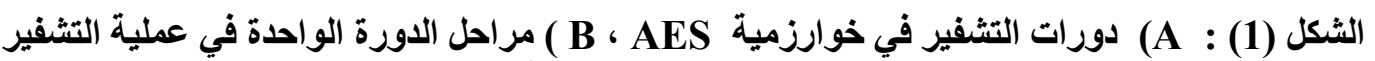

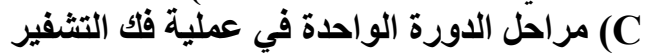

كما بمكن تصنيف هذه الخوارزمية إلى خمس أنماط تختلف في طريقة إدخال النص الأصلي المر اد تثفيره وكما يلي.

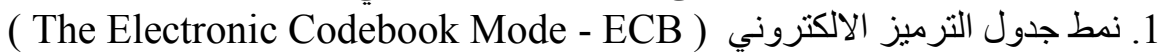

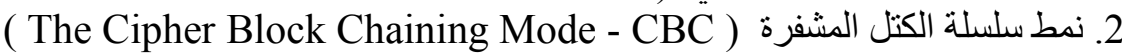
3. نمط التغذية الخفية بالنص المشفر ( The Cipher Feedback Mode - CFB ) 4. نمط التغذية الخلفية بالإخر اج ( The Output Feedback Mode -OFB )

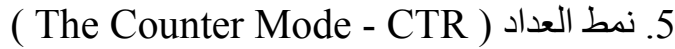


1.3

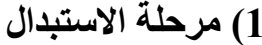

في هذه المرحلة يتم استبدال كل بايت يتم استقباله بقيمه أخرى كما موضح في الثكل(2)، وذلك بأخذ القيمة المر اد اد التئ

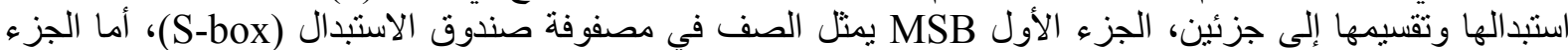

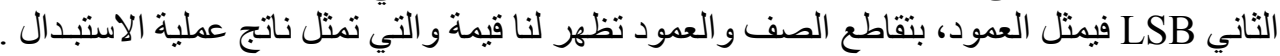

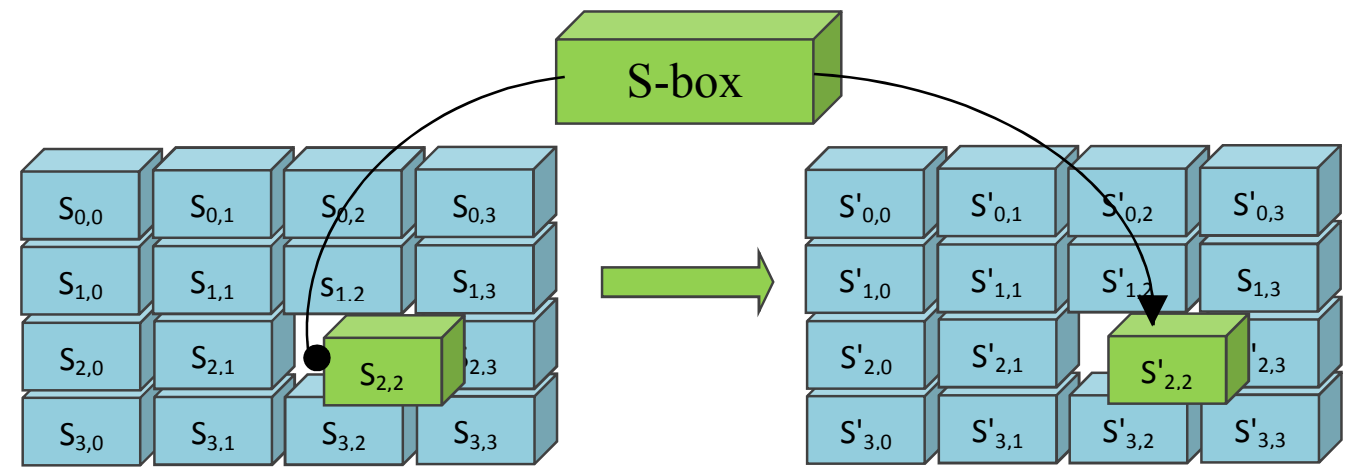

الشكل (2) : عملية الاستبدال

2) 2

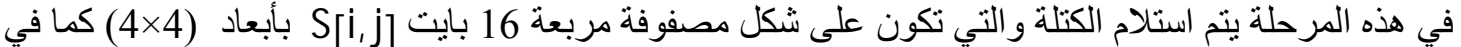

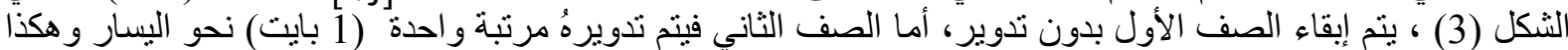

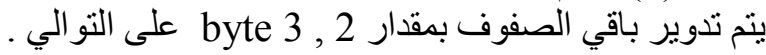

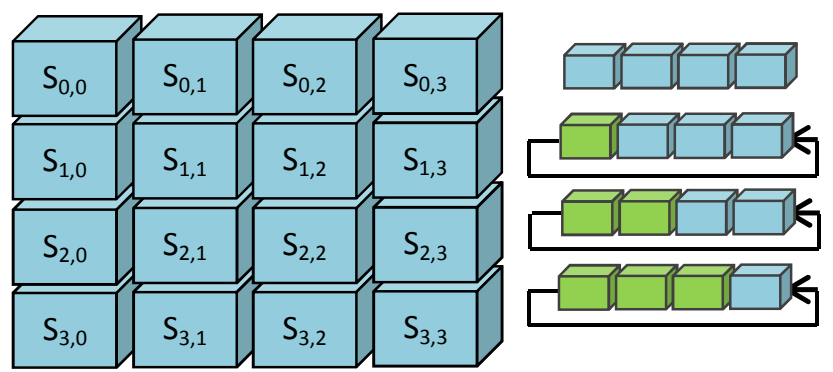

\begin{tabular}{|c|c|c|c|}
\hline$S_{0,0}$ & $S_{0,1}$ & $S_{0,2}$ & $S_{0,3}$ \\
\hline$S_{1,1}$ & $S_{1,2}$ & $S_{1,3}$ & $S_{1,0}$ \\
\hline$S_{2,2}$ & $S_{2,3}$ & $S_{2,0}$ & $S_{2,1}$ \\
\hline$S_{3,3}$ & $S_{3,0}$ & $S_{3,1}$ & $S_{3,2}$ \\
\hline
\end{tabular}

الشكل (3) : عملية التدوير

3) مرحلة مزج الأعمدة في هذه المرحلة يتم استبدال كل بايت في المصفوفة S[i,j] بقيم أخرى تعنمد على فئ قيم العناصر التابعة لنفس

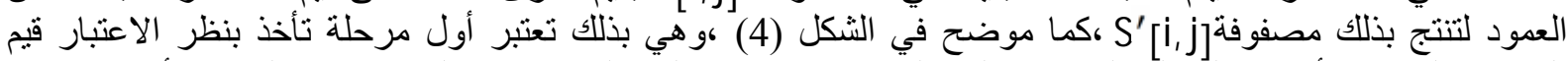
العناصر المجاورة أثناء عملية المعالجة وفق المعادله (1)، نوجد هذه المرحلة في جميع الدورات عدا الدئ الدورة الأخيرة.

$s_{0, \mathrm{j}}^{\prime}=\left(2 \cdot \mathrm{s}_{0, \mathrm{j}}\right) \oplus\left(3 \cdot \mathrm{s}_{1, \mathrm{j}}\right) \oplus \mathrm{s}_{2, \mathrm{j}} \oplus \mathrm{s}_{3, \mathrm{j}}$

$s_{1, \mathrm{j}}^{\prime}=\mathrm{s}_{0, \mathrm{j}} \oplus\left(2 \cdot \mathrm{s}_{1, \mathrm{j}}\right) \oplus\left(3 \bullet \mathrm{s}_{2, \mathrm{j}}\right) \oplus \mathrm{s}_{3, \mathrm{j}}$

$s_{2, \mathrm{j}}^{\prime}=\mathrm{s}_{0, \mathrm{j}} \oplus \mathrm{s}_{1, \mathrm{j}} \oplus\left(2 \cdot \mathrm{s}_{2, \mathrm{j}}\right) \oplus\left(3 \cdot \mathrm{s}_{3, \mathrm{j}}\right)$

$s_{3, \mathrm{j}}^{\prime}=\left(3 \bullet \mathrm{s}_{0, \mathrm{j}}\right) \oplus \mathrm{s}_{1, \mathrm{j}} \oplus \mathrm{s}_{2, \mathrm{j}} \oplus\left(2 \bullet \mathrm{s}_{3, \mathrm{j}}\right)$ 


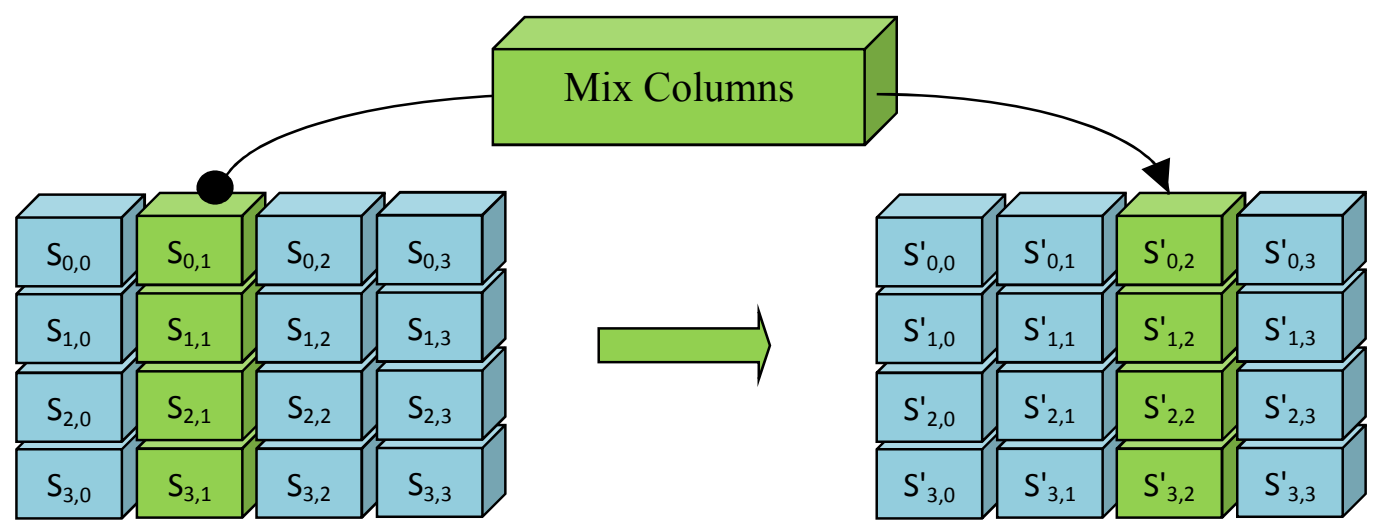

الشكل (4) : مرحلة مزج الأعمدة

4) مرحلة إضافة مفتاح الدورة

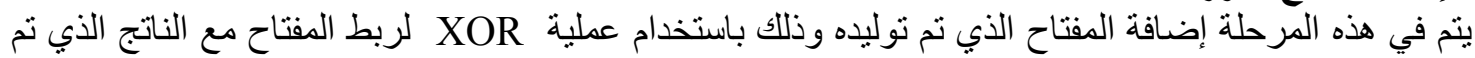

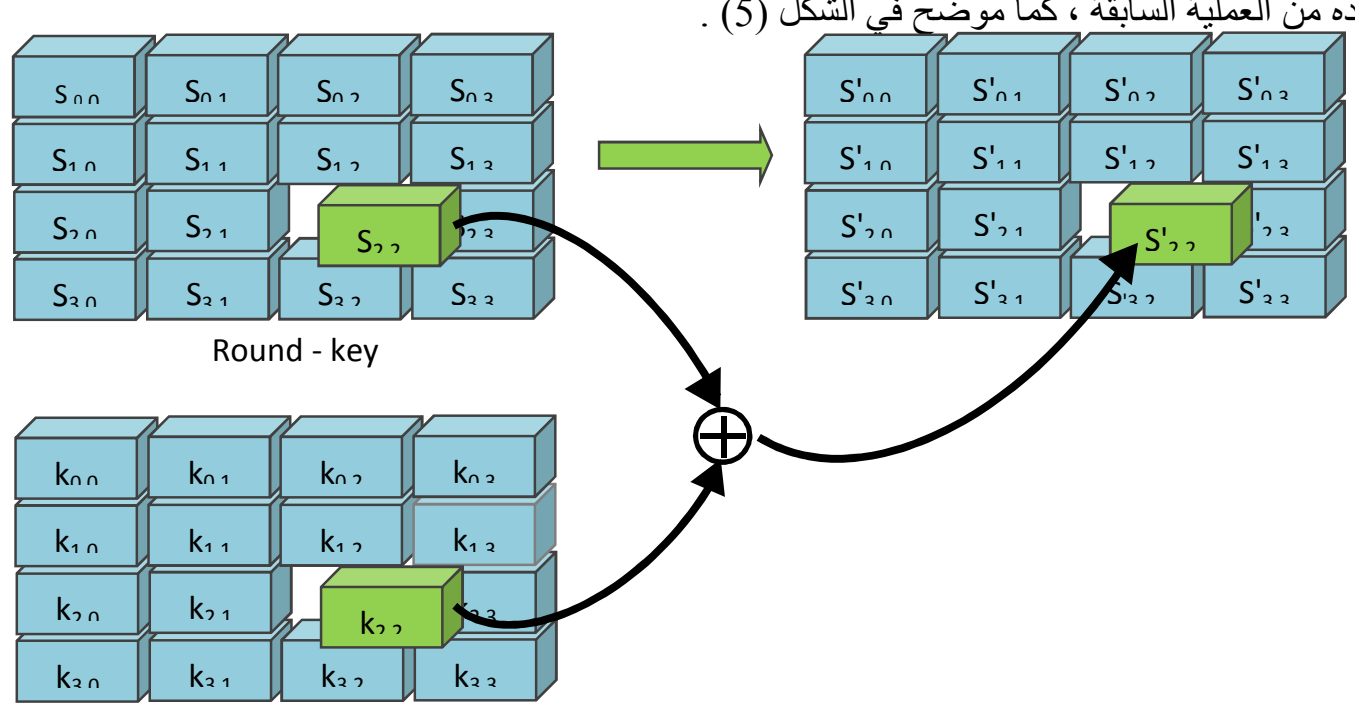

الثكل (5) : عملية إضافة المفتاح

4- مولد التسلسل شبه العشوائي (Pseudo Random Sequence Generator - PRSG)

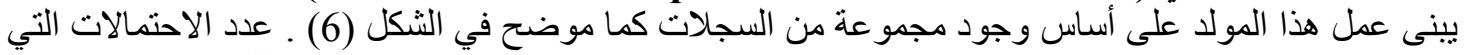

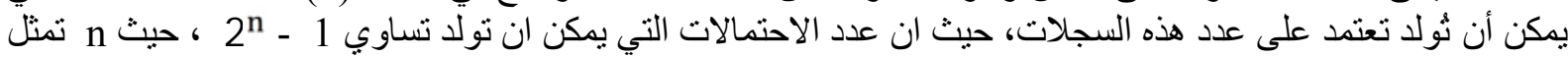

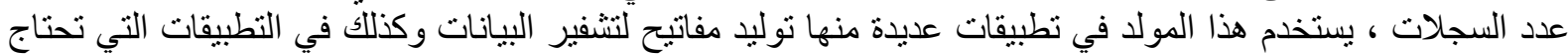

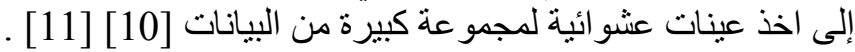

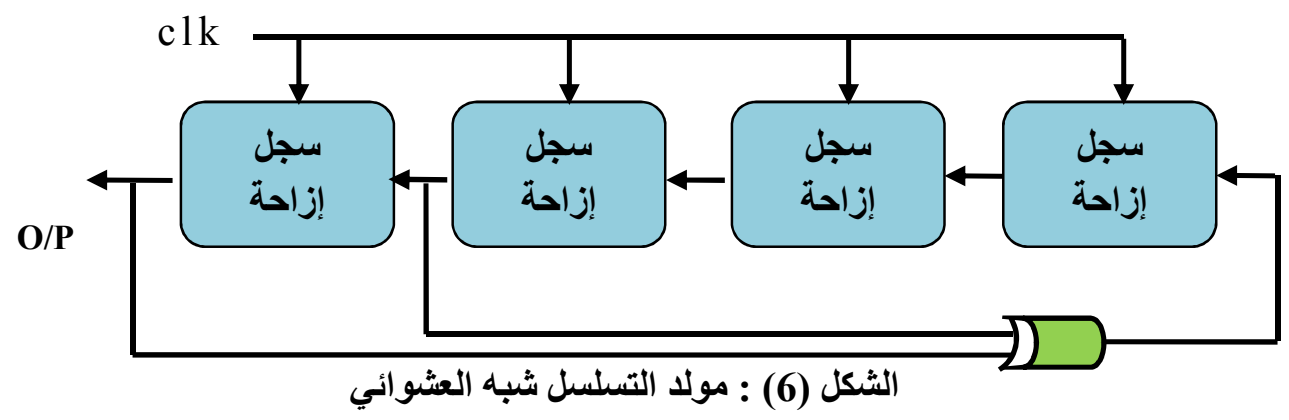




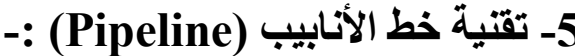

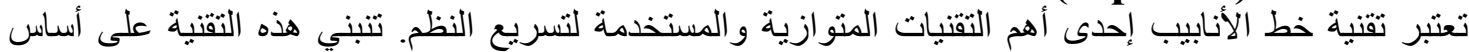

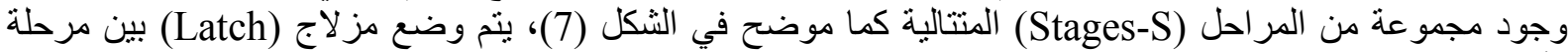

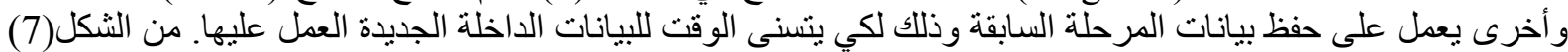
يمكن ملاحظة ان التقنية تعتمد على نابض (Clock) مشترك و الذي يعمل على السيطرة على سير البيانات على طول مسلى التيار

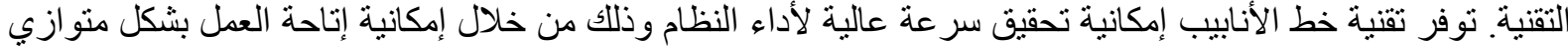

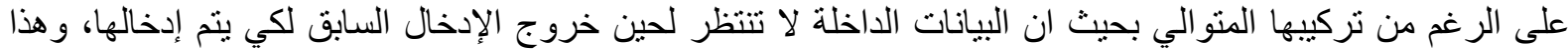

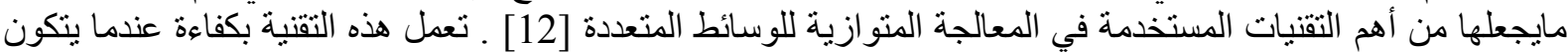
الإدخال من عدد كبير من المهام المتتالية .

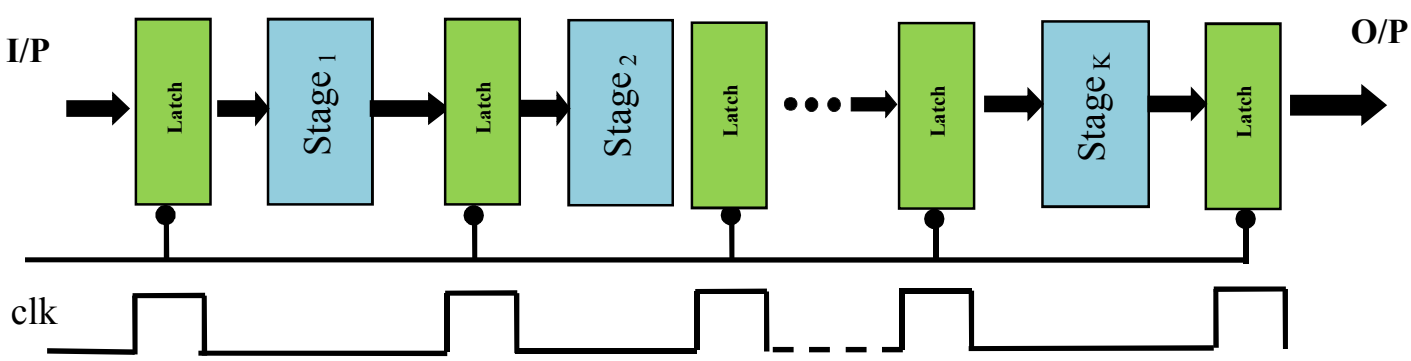

الشكل (7) مراحل تقنية خط الأنابيب

6- 6ـاليب تنفيذ المعماريات المصممة

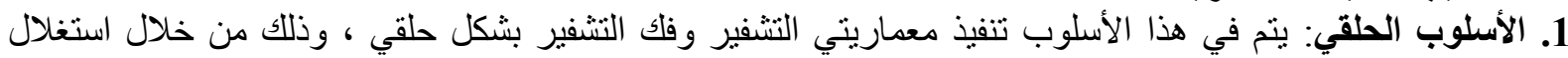

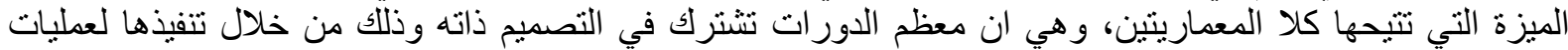

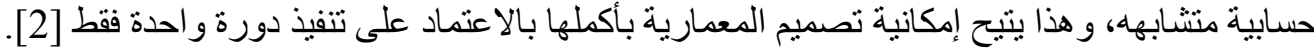

2. أسلوب خط الأنابيب: في هذا الأسلوب يتم تتفيذ المعمارية بالاعتماد على تقنية خط الأنابيب، وذلك باعتبار كل دورة من دورات الـ AES عبارة عن مرحلة من مر احل تقنية خط الأنابيب [2].

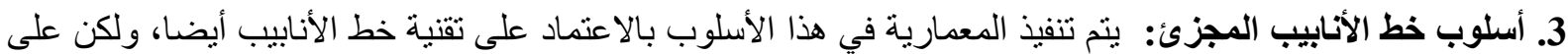

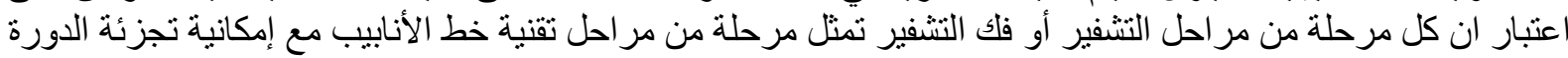

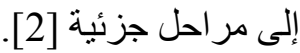

\section{7- 7 المعماريات المصممة}

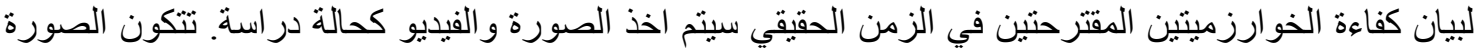

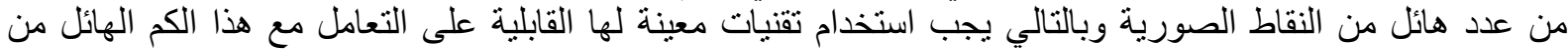

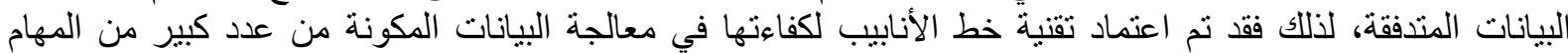

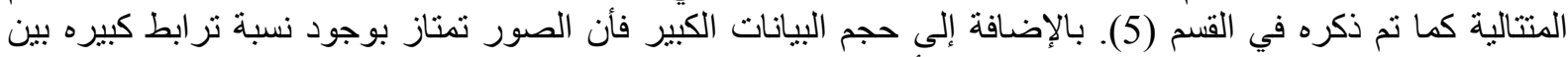

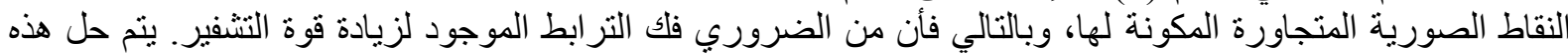

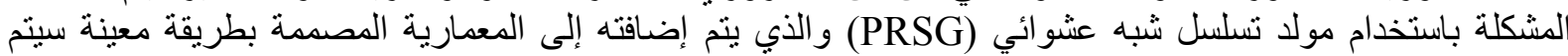

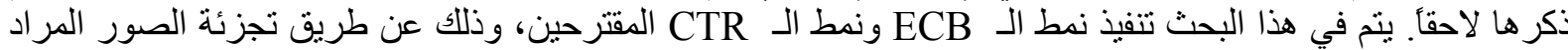

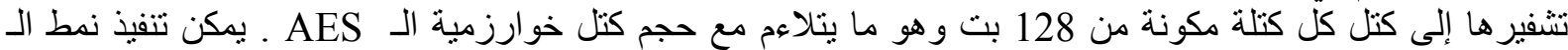
(ECB) و (CTR) بشكل منو ازي وذللك لعدم اعتماد معالجة (تشفير ) الكتلة الحالية على ناتج الكتلة السابقة.

1.7 في هذا المقترح يتم إدخال بيانات الصولى الصورة إلى الخوارزمية المقترحة كما موضح في الثنكل (8)، بعد دخول

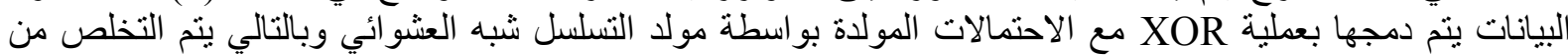
المشكلة الأولى التي يتميز بها هذا النوع من البيانات ( الترابط بين النقاط الصورية بلئ ) بعد ذلك ينت إدخال كل ناتج من العملية 


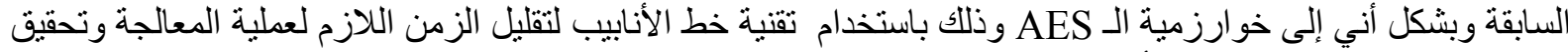

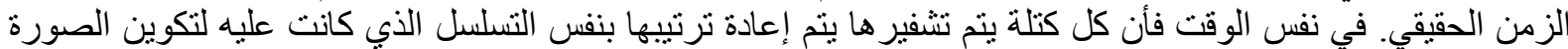

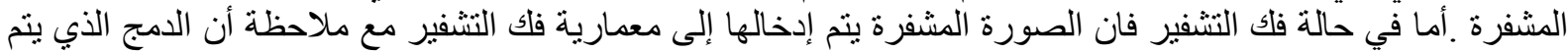

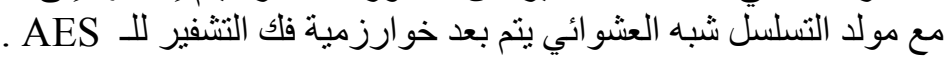

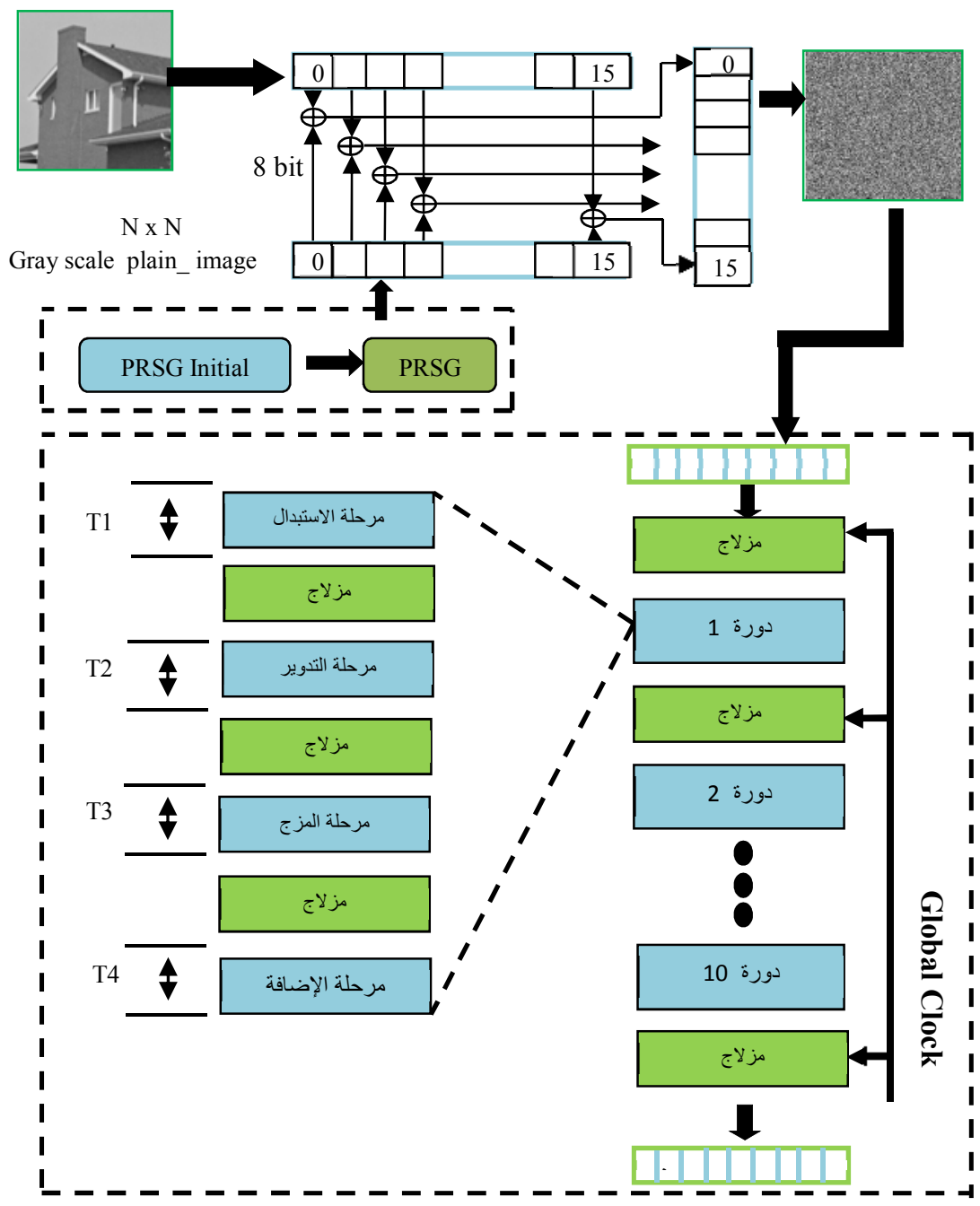

الثكل (8) : آلية عمل المعمارية المقترحة الأولى

2.7

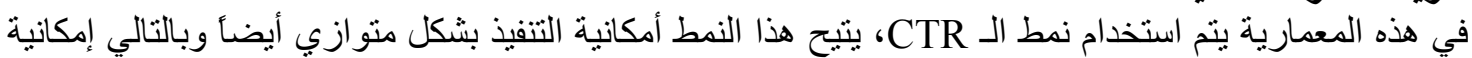

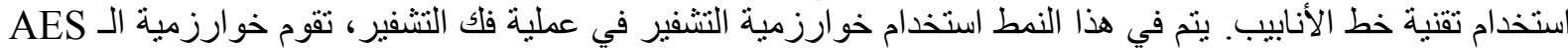

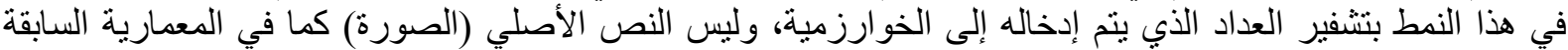

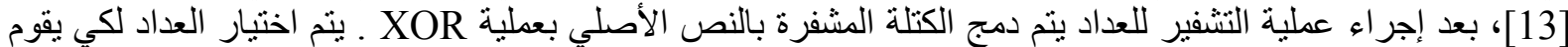

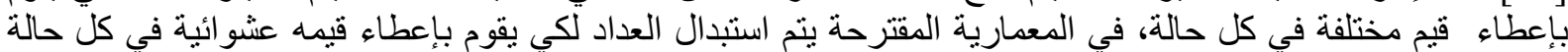

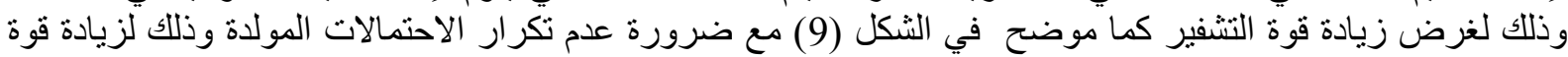




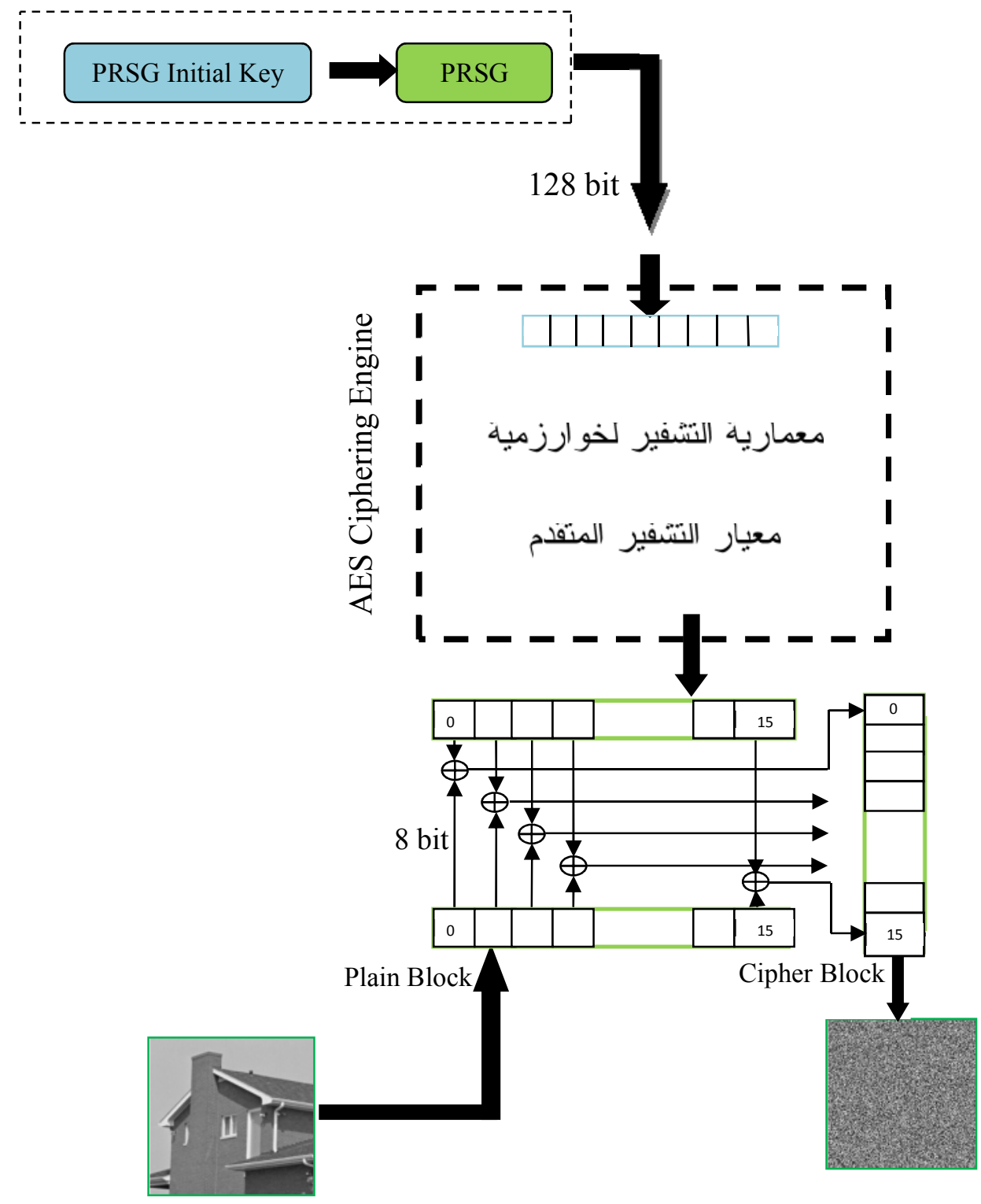

الثكل (9) : آلية عمل معمارية التثفير وفك التثفير المقترحة الثانية

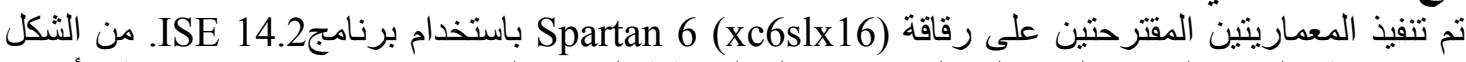

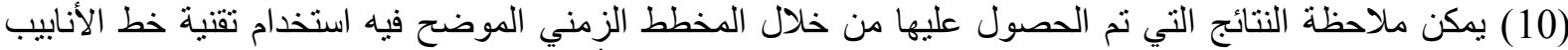

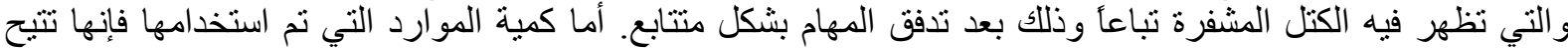
إمكانية تركيب التصاميم على الثريحة المذكورة و التي يمكن إدراجها في الجدول (1) . 


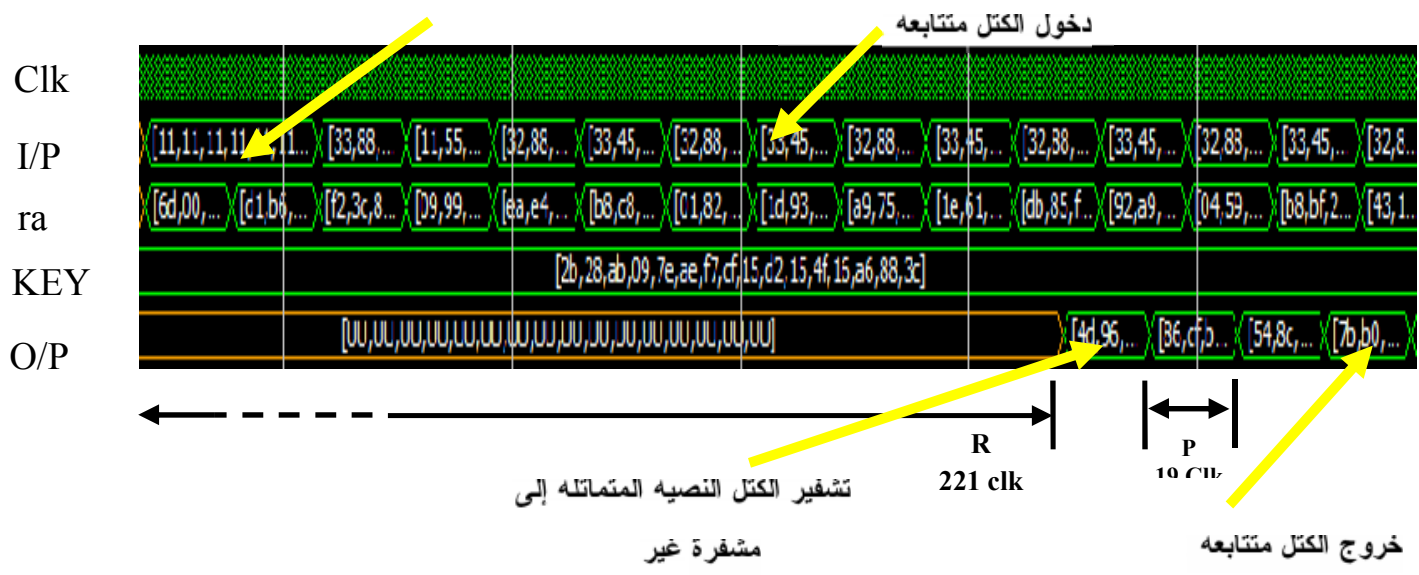

الثكل (10) : نتائج المحاكاة للمعماريتين المقترحة الأولى و الثانية . .

جدول (1) : يوضح كمية الموارد المستخدمة لبناء المعماريتين

\begin{tabular}{|c|c|c|c|c|c|}
\hline \multicolumn{7}{|c|}{ Device Utilization Summary (estimated values) } \\
\hline Logic Utilization & $\begin{array}{c}\text { Used/ENC1/ } \\
\text { ENC2/DEC2 } 2\end{array}$ & $\begin{array}{c}\text { Used/ } \\
\text { DEC1 }\end{array}$ & Available & $\begin{array}{c}\text { Utilization/ENC1 } \\
\text { / ENC2/DEC2 }\end{array}$ & $\begin{array}{c}\text { Utilization/ } \\
\text { DEC1 }\end{array}$ \\
\hline Number of Slice Registers & 6874 & 10,946 & 18224 & $37 \%$ & $60 \%$ \\
\hline Number of Slice LUTs & 5307 & 6,904 & 9112 & $58 \%$ & $75 \%$ \\
\hline Number of fully used LUT-FFpairs & 3308 & 5,669 & 8873 & $37 \%$ & $65 \%$ \\
\hline Number of Block RAM/FIFO & 1 & 1 & 32 & $3 \%$ & $3 \%$ \\
\hline Maximum Frequency & \multicolumn{7}{|l}{$158.234 \mathrm{MHz}$} \\
\hline
\end{tabular}

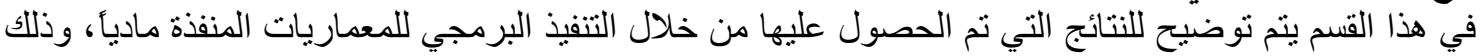

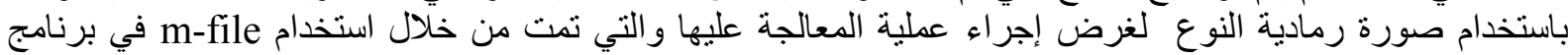

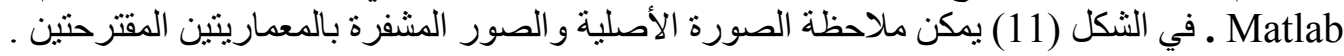

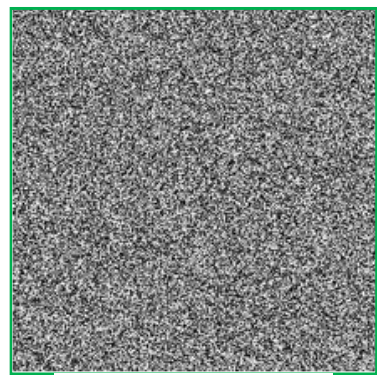

$\mathrm{C}$

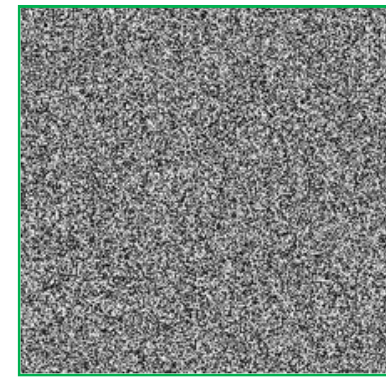

$\mathrm{B}$

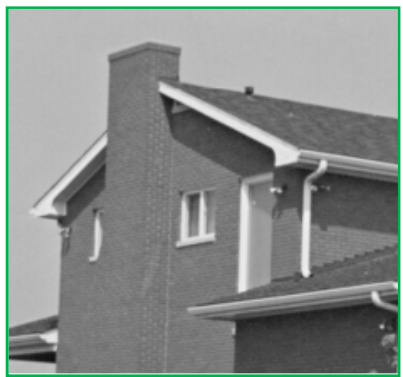

A

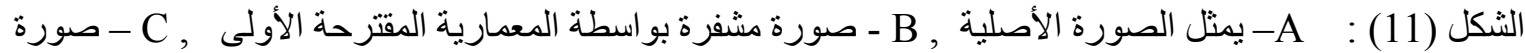
مشفرة بو اسطة المعمارية المقترحة الثانية

\subsection{8 نتائج التحليل الإحصائي

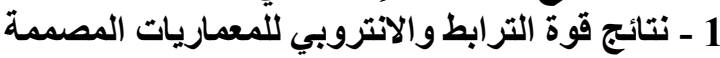

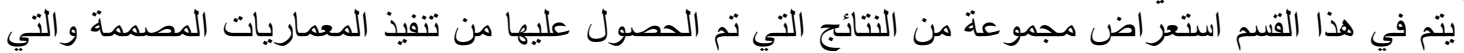

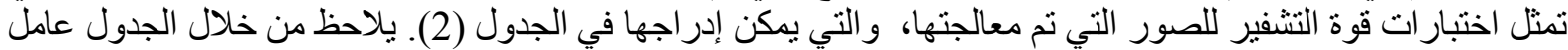

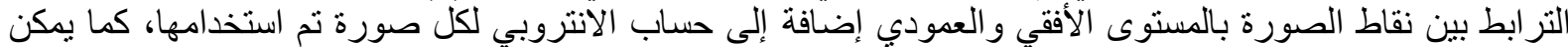
ملاحظة التحسن في عامل الترابط من خلال تقليل الترابط بين النقاط الصورية وذللك عند استخدام مولد التسلسل شبه 
العشو ائي، بالإضافة إلى ملاحظة التحسن (زيادة) الانتروبي والتي تعتبر مقياس للعشو ائبة، حيث تكون الانتروبي مساوية

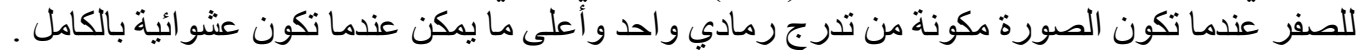
الجدول(2) : قياس قوة الترابطوالانتروبي .

\begin{tabular}{|c|c|c|c|}
\hline \multirow{2}{*}{ Image } & \multicolumn{2}{|c|}{ Correlation Analysis } & \multirow{2}{*}{$\begin{array}{c}\text { Entropy } \\
\text { value }\end{array}$} \\
\cline { 2 - 3 } & Vertical & Horizontal & 6.4971 \\
\hline الصورة الأصلية & 0.96528 & 0.97807 & 7.9971 \\
\hline ECB & 0.0072677 & 0.00056767 & 7.9969 \\
\hline 1 المقترحتر & 0.0026851 & -0.00074783 & 7.9969 \\
\hline CTR & 0.00023802 & -0.0023204 & 7.9974 \\
\hline 2 المقترحة & -0.00018942 & 0.0019987 & \\
\hline
\end{tabular}

2نتائج المدرج التكراري (Histogram) للمعماريات المصممة

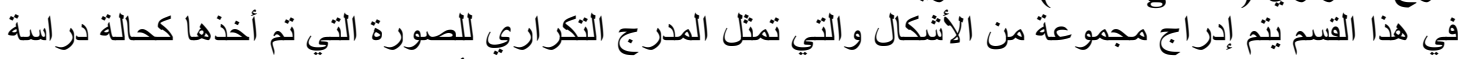

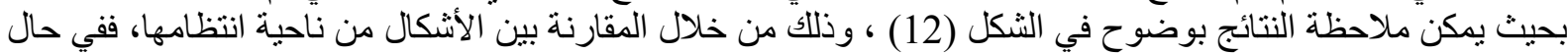

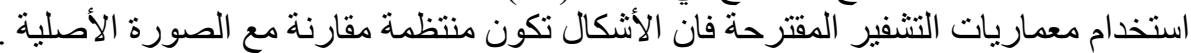

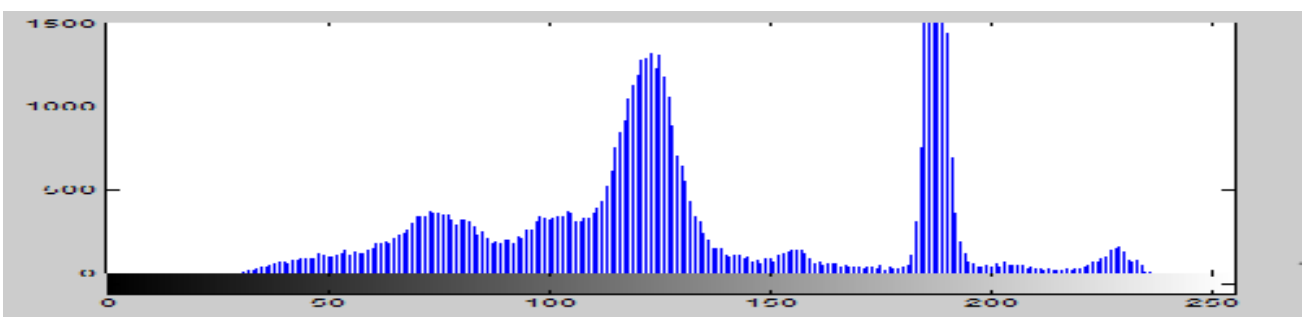

الصورة الأصلية

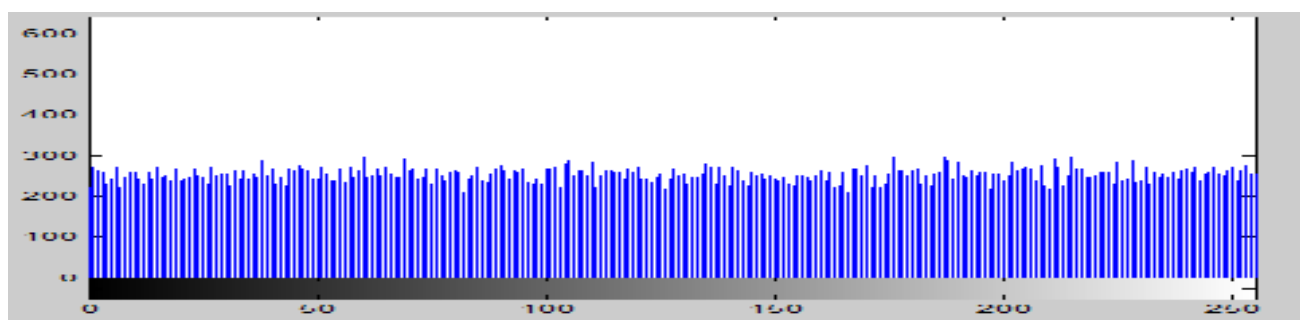

صورة مشفرة بواسطة المعمارية المقترحة الأولى

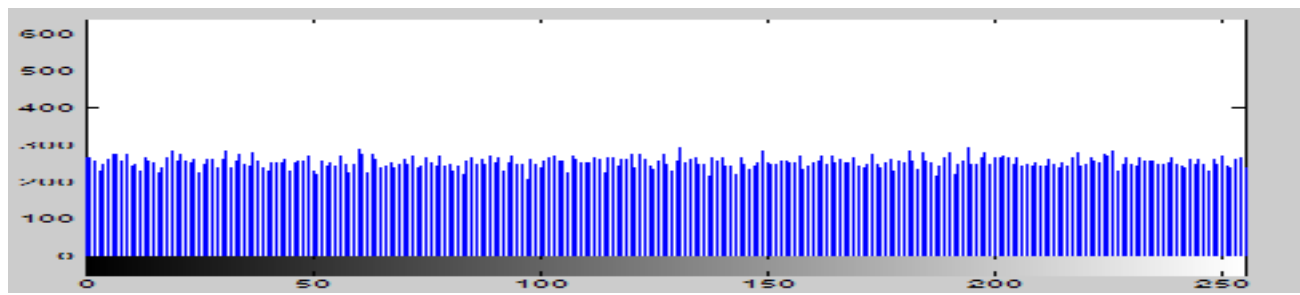

صورة مشفرة بو اسطة المعمارية المقترحة الثانية

الثكل (12) : المدرج التكراري لمجموعة من الصور 
يمكن حساب الزمن المستغرق لتشفير صورة بحجم (32 x 64) لكلا المعماريتين وذلك باستخدام المعادلة (2) .

Time of Pipeline $($ Image $)=\frac{C}{F}=\frac{((B-1) * P)+R}{F}$

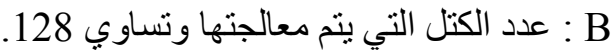

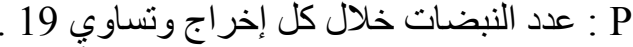

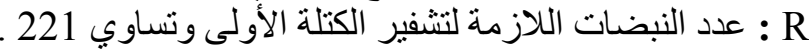

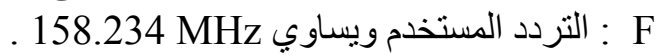

Time of Pipeline $($ Image $)=\{[(((32 * 64) / 16)-1) * 19]+221\} / 158.234 \mathrm{MHz}$

$$
=16.64 \mu \mathrm{s}
$$

أما التسارع (Speedup) فيمكن حسابه وفق المعادلة (3) .

Speedup $=\frac{\text { Time of Non }- \text { Pipeline }}{\text { Time of Pipeline }}$

$\because$ Time of Non-Pipeline $=\frac{B * R}{F}$

Time of Non - Pipeline $($ Image $)=\frac{128 * 221}{158.234}=178.77 \mu \mathrm{sec}$

$\therefore$ Speedup $=\frac{178.77}{16.64}=10.74$

من الملاحظ ان كلا المعماريتين تم تنفيذها وفق منطلبات الزمن الحقيقي وان الزمن الدستغرق اقل بكثير من الزمن اللازم

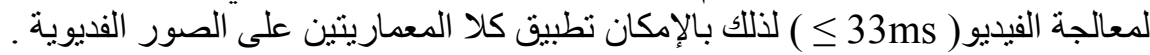

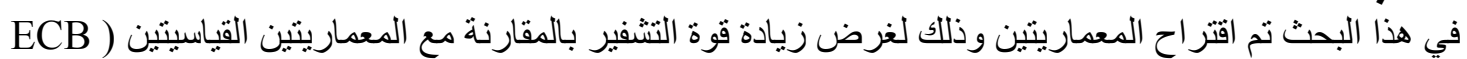

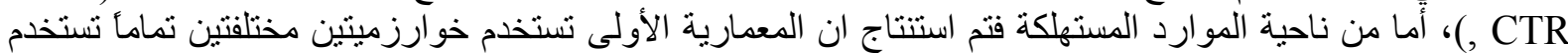

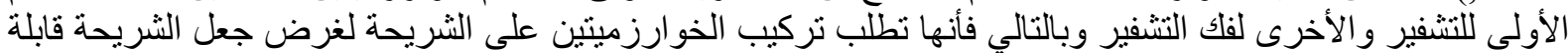

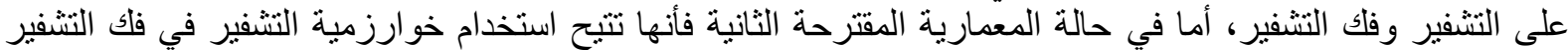

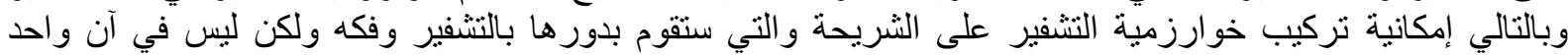
وبالتالي اختصار الموارد اللازمة للتنفيذ. أما بالنسبة للتنفيذ في الزمن الحقيقي فأن كلا المعماريتين تم تتفيذهما ضمن

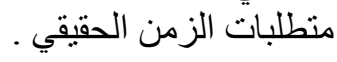

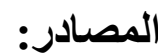

[1] S. Lian, "Multimedia Content Encryption" , (c) by Taylor \& Francis Group, LLC, International Standard Book Number-13: 978-1-4200-6527-5 (Hardcover), 2009 .

[2] X. Zhang, k. Parhi," Implementation approaches for the advanced encryption standard algorithm ", Journal: IEEE Circuits and Systems Magazine ISSN: 1531636x , 2002.

[3] S. Yoo, D. Kotturi, W.Pan, J. Blizzard, " An AES crypto chip using a high-speed parallel pipelined architecture ", Microprocessors and Microsystems 29.7 : 317-326. , 2005.

[4] F.Shamsulddin, "On the security of Bitmap Images using Scrambling based Encryption Method", Journal of Engineering and Development, Vol. 13, No. 3,September, ISSN 1813-7822 2009 . 
[5] R. Paul, S. Saha, S. Sau, A. Chakrabarti, "Design and implementation of real time AES128 on real time operating system for multiple FPGA communication", arXiv preprint arXiv:1205.2153, 2012 ..

[6] F. Bin , Muhay, "Chaotic and AES cryptosystem for satellite imagery ", Journal: Telecommunication Systems ISSN: 10184864, Volume:52 Issue:2 Pages:573-581 Provider: Springer, DOI:10.1007/s11235-011-9462, 2013.

[7] A.Mathur,"A Research paper: An ASCII value base data encryption algorithm and its comparison with other symmetric data encryption algorithms", International Journal 4 on Computer Science and Engineering (IJCSE), 2012 .

[8] J. Daemen, V.Rijmen, "The Design of Rijndael : AES - The Advanced Encryption Standard", copyright (C) ISBN 3-540-42580-2 Springer-Verlag Berlin Heidelberg New York .

[9] A. Deshpande, M. Deshpande, D. Kayatanavar," FPGA Implementation of AES Encryption and Decryption" , Control, Automation, Communication and Energy Conservation, INCACEC 2009. International Conference on. IEEE, 2009 .

[10] "Efficient Shift Registers, LFSR Counters ,and Long Pseudo-Random Sequence Generators", URL: http://www.xilinx.Com/bvdocs/appnotes/xapp052 , XAPP 052 July 7,1996 (Version 1.1) .

[11] A. Kumar, P. Rajput ,et al ," Design Of Multi Bit LFSR PNRG And Performance Comparison On FPGA Using VHDL", International Journal of Advances in Engineering \& Technology, March 2012. ISSN: 2231-1963 .

[12] K.Hwang , "Advanced Computer Architecture ",ISBN 0-07-031622-8, Copyright (C) by McGraw-Hill, Inc, 1993.

[13] M. Dworkin, "Recommendation for Block Cipher Modes of Operation", No. NIST-SP800-38A. National Inst of Standards and Technology Gaithersburg MD Computer Security DIV, 2001.

تم اجراء البحث في كلية ألهندة = جامعة ألموصل 